\title{
Cultivation of Treponema pallidum in subcutaneous chambers implanted in golden hamsters
}

\author{
G. D. MORRISON,* S. I. EGGLESTONE,† AND J. L. NORTHWOOD \\ From the *Venereology Department and $\ddagger$ Public Health and Microbiology Laboratory, Plymouth \\ General Hospital; and the School of Environmental Sciences, Plymouth Polytechnic, Plymouth
}

SUMMARY Treponema pallidum was succesșfully grown in subcutaneous chambers in golden hamsters. Abnormally long forms of $T$. pallidum were found in these chambers. The cause of this abnormality may possibly be due to their being coated with antibody which could inhibit cell division or render the organism avirulent or both. $T$. pallidum was successfully passaged between hamsters but with a possible diminution in virulence.

\section{Introduction}

The technique of growing Treponema pallidum in a chamber implanted in a host animal was described by Levaditi and McKintosh (1908). These authors used collodion sacs which were inserted into the peritoneal cavities of cynomolgous monkeys and rabbits. Secondary infection in the sacs rendered the technique unusable. The method was improved by Arko (1973), who used a plastic practice golf ball to form a subcutaneous chamber in the back of a rabbit. Tight and Perkins (1976) used this system to study the growth of $T$. pallidum in the rabbit. They also showed that the administration of corticosteroids increased the yield in the chamber but that the administration of oxisuran, which inhibits cellmeditated immunity, did not do so. A disadvantage of these chambers in the rabbit is the formation of chancres at the site of entry of needles used to sample the chamber contents, with the risk of the overlying skin breaking down. Rathlev (1973) used chambers made of small lengths of polythene tubing implanted under the skin of guinea pigs in which $T$. pallidum could be grown.

As we were interested in the development of a system which might isolate $T$. pallidum from body fluids, we originally tried this technique in albino rats but were unable to grow $T$. pallidum in them. Another drawback was the development of fibrosarcomas around the chambers, presumably due to carcinogens in the plastic. We then attempted the same technique in golden hamsters (Mesocricetus

Address for reprints: Dr G. D. Morrison, Treatment Clinic, Plymouth General Hospital, Freedom Fields, Plymouth, Devon

Received for publication 19 March 1979 auratus) and report here the successful growth in, and passage between, these chambers. The golden hamster was chosen because it is known not to have a naturally occurring treponemal infection and also because Turner and Hollander (1957) had shown that hamsters can carry $T$. pallidum over long periods.

\section{Materials and methods}

INSERTION OF CHAMBERS

Golden hamsters were anaesthetised with a mixture of halothane and air and placed prone on the operating table. The upper half of the back was clipped free of hair and the skin thoroughly disinfected. A midline incision, approximately $1-\mathrm{cm}$ long, was made just posterior to the scapulae. Spencer Wells forceps were passed subcutaneously in a posterolateral direction to form a pocket in each flank and a sterile chamber inserted in each pocket using an aseptic technique. The skin incision was closed with Michel clips and the wound sprayed with a clear plastic dressing (Nobecutane, Astra Ltd.). The chambers were made of $2 \cdot 5-\mathrm{cm}$ lengths of Eschman polyvinyl chloride conductive tubing, internal bore $0.5 \mathrm{~cm}$, and sterilised by autoclaving. The total volume of these chambers is $0.5 \mathrm{ml}$ but the recoverable volume is $0.3 \mathrm{ml}$. After seven days the clips were removed under a light anaesthetic, and all subsequent procedures were performed on anaesthetised animals to prevent the operator being bitten. The hamsters were not used for at least 14 days, during which time the chambers filled with a clear fluid which on electrophoretic analysis showed only albumen and $\beta$-globulins to be present (Fig. 1). The animals were housed individually in cages at an environmental temperature of $60-70^{\circ} \mathrm{F}$, fed on an 


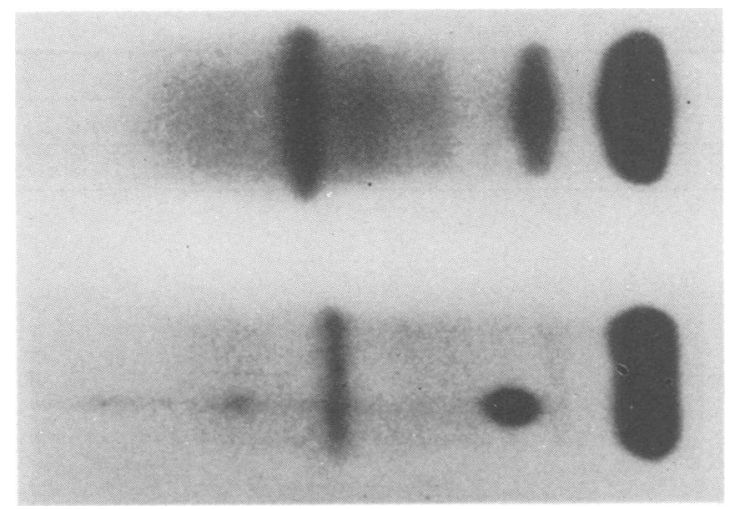

Fig. 1 Electrophoretic strips: upper strip = serum; lower strip = chamber fluid

antibiotic-free, commercially prepared, rodent food and given an unlimited amount of water.

\section{SOURCE OF T. PALLIDUM}

An initial intratesticular inoculation of $T$. pallidum (Nichols strain) in a rabbit produced the typical orchitis. The rabbit was killed and the testes removed. The $T$. pallidum was extracted from the testicular tissue as described by Wilkinson (1972). The treponemes were suspended in $\mathrm{pH} 7.4$ phosphate-buffered saline and diluted to give a count of approximately 20 organisms per high power field using a $\times 100$ oil immersion objective and darkground illumination.

\section{INOCULATION}

Three experimental groups of hamsters (A, B, and C) were used and $0.02 \mathrm{ml}$ of the treponeme suspension was injected into the chamber of each animal. The skin over one end was disinfected with $70 \%$ alcohol, and a fine needle $(25 \mathrm{G})$ was inserted. Only very small quantities were withdrawn (enough to give one drop on a microscope slide) to avoid emptying the chamber. In addition to the treponeme suspension the hamsters in group B received an intramuscular injection of $0.02 \mathrm{ml}$ methylprednisolone (Depo Medrone, Upjohn) in their thighs and group $C$ an intramuscular injection of $0.02 \mathrm{ml}$ of a long-acting triamcinolone (Kenalog, Squibb) to lengthen the duration of the immunosuppressive activity.

In a fourth group of hamsters, which were not inoculated with treponemes, chamber fluid was examined before and after treatment with long-acting triamcinolone to determine if naturally occurring treponeme-like forms occurred in the chambers.

\section{MICROSCOPY AND SEROLOGY}

Samples of fluid were withdrawn from the chambers at weekly intervals. One drop was placed on a slide and 20 fields were counted using darkground illumination and a $\times 100$ objective. Passages between hamsters were performed by injecting $0.01 \mathrm{ml}$ into the chamber of the recipient hamster.

Samples of chamber fluid were further examined by silver-staining, using Dunoyer's modification of Dieterle's method (Collart et al., 1962) and by fluorescent antibody staining using an indirect method with known positive human serum (Venereal Disease Research (VDRL) test titre 1/32) from a patient with secondary syphilis and fluorescein conjugated anti-human globulin (Wellcome Laboratories Ltd).

Serological testing of chamber fluid and blood from the hamsters was performed when the animals were killed. We found that cardiac puncture caused too high a fatality rate to be performed more than once. The tests used were the $T$. pallidum haemagglutination assay (TPHA), the VDRL test, and the fluorescent treponemal antibody (FTA) test using anti-hamster globulin (Difco Laboratories) with Wellcome FTA antigen.

\section{Results}

The hamsters tolerated both the chambers and the repeated anaesthetics with no apparent ill effects. Post-mortem examination of hamsters whose chambers had been inoculated with $T$. pallidum did not show any lymphadenopathy, and histological examination of the skin overlying the end of the chamber showed no inflammatory response but only fibrous tissue with iron deposits, presumably from bleeding when the chamber was inserted.

The results of the attempts to grow $T$. pallidum in the chambers is shown in Table 1 and 2 and subsequent passage experiments in Table 3. Motile treponemes of normal length seen in the chamber fluid had the characteristic corkscrew morphology of T. pallidum (Fig. 2). Double-length organisms about to divide were commonly seen. In addition organisms between three and eight times their normal length were found. They showed diminished motility and less pronounced undulations (Fig. 3). These originally appeared eight weeks after the initial inoculation in hamsters treated with triamcinolone (group C) but not in those initially treated with methylprednisolone (group B) or untreated controls (group A). Subsequent passages from group C animals on all further occasions have shown both short and long forms in the chambers of the recipient hamsters. The ratio between these two forms was not constant, and there were times when the long forms disappeared and only normal-length forms were seen. Both normal and long forms showed positive fluorescent-antibody staining. Uninoculated 
Table 1 Results of inoculation of hamster chambers

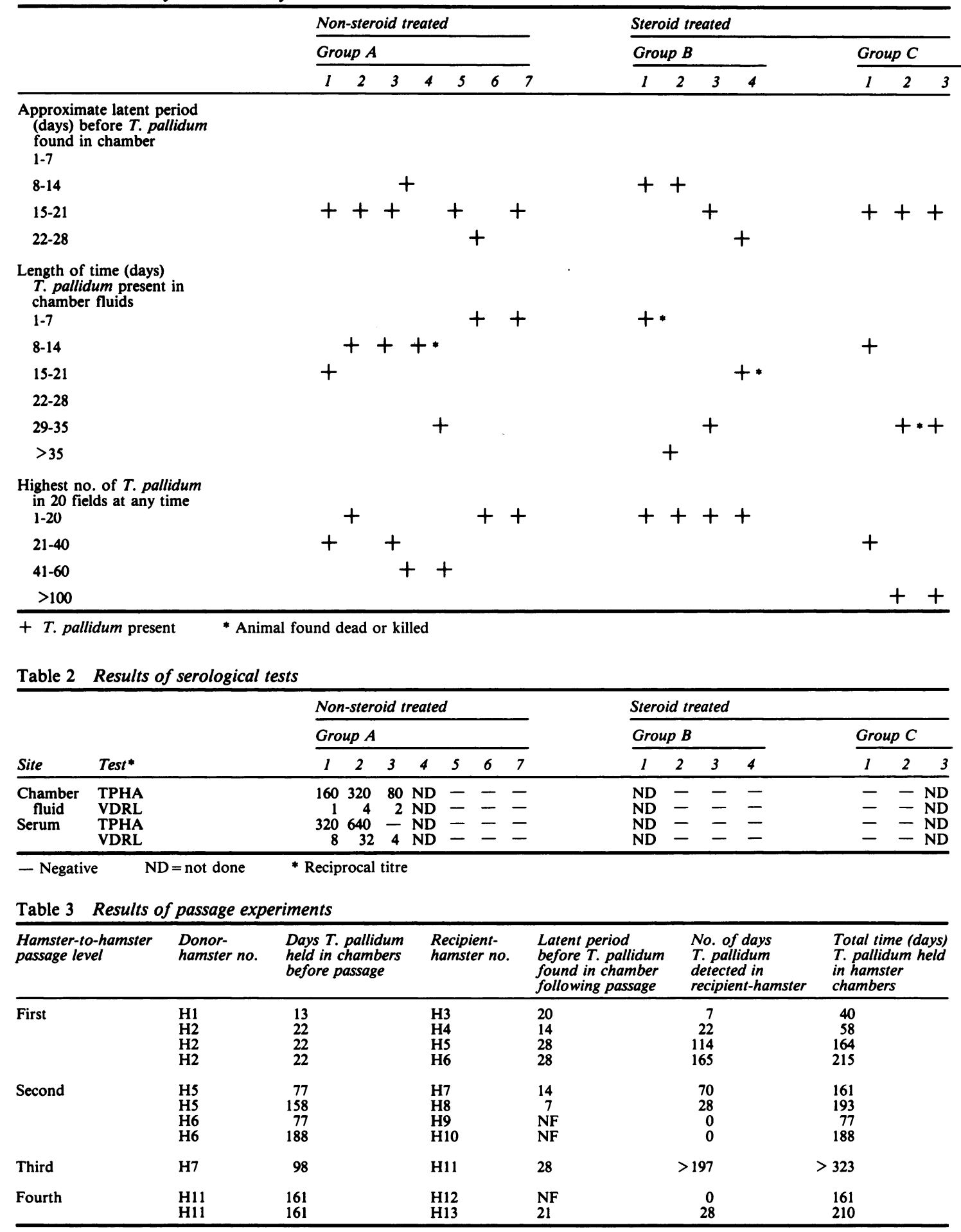

Table 2 Results of serological tests

.




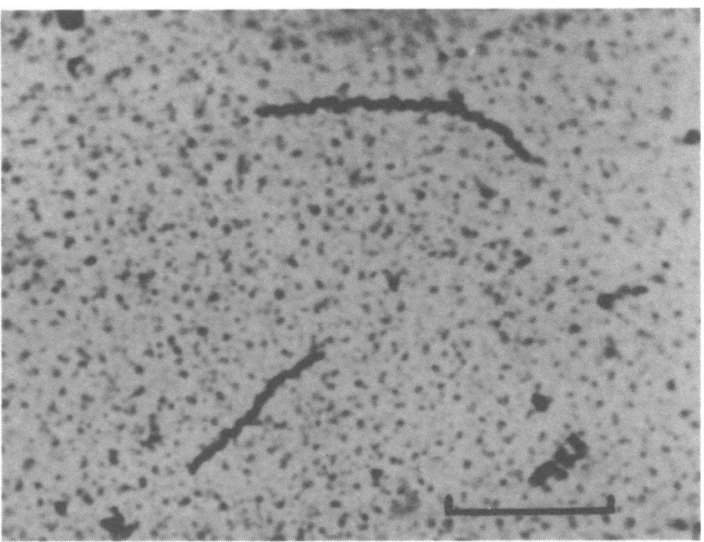

Fig. 2 Normal length treponemes as seen in chamber fluid. (Stained by Dunoyer's modification of Dieterle's method; internal scale marker $=10 \mu$ )

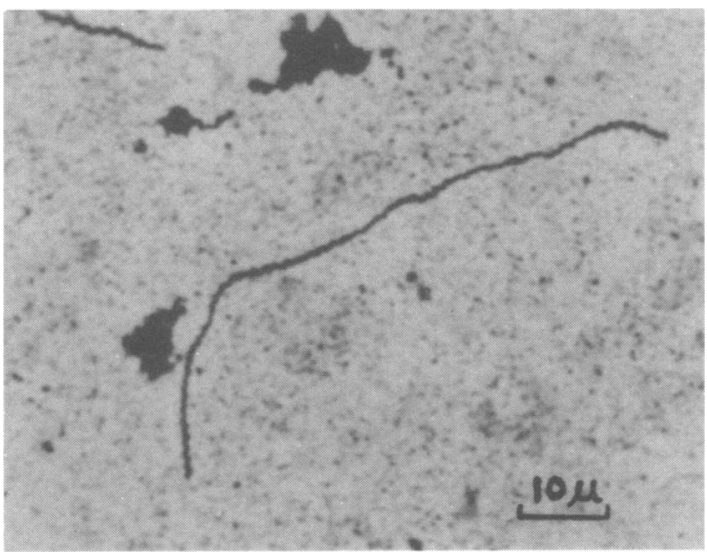

Fig. 3 Long form of treponemes as seen in chamber fluid. (Stained by Dunoyer's modification of Dieterle's method; internal scale marker $=10 \mu$ )

hamsters, whether or not they had been treated with steroids, showed no organisms resembling treponemes in their chamber fluids.

Although the size of the drop of chamber fluid examined on different occasions varied slightly, the treponemes seemed to increase in numbers after a latent period during which no $T$. pallidum could be seen.

Chamber fluid containing long and short forms of $T$. pallidum was inoculated under the shaved neck of two rabbits with known negative serology; neither rabbit developed a chancre nor did it convert its serology during the subsequent three months.
Furthermore, direct-staining of the long forms using Difco anti-hamster fluorescein conjugate showed weak fluorescence.

\section{Discussion}

The hamster is known not to have a naturally occurring treponemal infection, and we were also unable to demonstrate treponeme-like forms in the chamber fluids of uninoculated controls with or without the administration of steroids. However, intradermal inoculation of hamsters with $T$. pallidum produces a generalised lymphadenitis with numerous treponemes (Turner and Hollander, 1957). As these changes were not seen in inoculated hamsters in the present study, it would suggest that encapsulation of the chamber takes place. The encapsulation could also explain, owing to lack of antigenic stimulus, the low systemic immune response observed.

The chamber system used was shown to support the growth of $T$. pallidum and it also showed that passage between hamster chambers could be achieved. However, many of the treponemes were clearly different in their morphology and virulence. Furthermore, the long forms showed some evidence of being coated with antibody, and it is possible that this antibody could inhibit cell division or render the organism avirulent or both. One cannot rule out the possibility that the organisms may have encountered deficiencies in essential metabolites while growing in the chambers, which may have affected their morphology and virulence.

We cannot explain at the present time the apparent inconsistencies of the immunological responses in the non-steroid treated group. Work is continuing in an attempt to elucidate these problems.

We wish to thank Dr A. E. Wilkinson and Mr G. Lavender of the Venereal Disease Reference Laboratory for supplying the Treponema pallidum, Dr P. J. L. Sequeira for helpful advice, Mrs M. Ashworth for attending to the welfare of the animals, Dr P. D. Meers and Dr G. Churcher for making available the facilities of the Plymouth Laboratory of the Public Health Laboratory Service, Dr J. Went for the electrophoretic studies, Dr A. C. Hunt for histological examinations, and Miss C. Ellis for typing the manuscript.

\section{References}

Arko, R. J. (1972). Neisseria gonorrhoeae: experimental infection of laboratory animals. Science, 177, 1200-1201.

Collart, P., Borel, L. J., and Durel, P. (1962). Etude de l'action de la pénicilline dans la syphilis tardive. Persistence du tréponème pâle après traitement. Annales de l'Institut Pasteur, 102, 596-615. 
Levaditi, C. and McKintosh, J. (1907). Contribution à l'étude de la culture de Treponema pallidum. Annales de l'Institut Pasteur, 21 , 784-797.

Rathlev, T. (1973). Cultivation of Treponema pallidum in chambers surgically implanted in experimental animals. Acta Pathologica et Microbiologica Scandinavica, 81 (2), 269-271.

Tight, R. R. and Perkins, R. L. (1976). Treponema pallidum infection in subcutaneous polyethylene chambers in rabbits. Infection and Immunity, 13, 1606-1612.
Turner, T. B. and Hollander D. H. (1957). Biology of the treponematoses. World Health Organisation Monograph Series No. 35. WHO: Geneva.

Wilkinson, A. E., Taylor, C. E. D., McSwiggan, D. A., Turner, G. C., Rycroft, J. A., and Lowe, G. H. (1972). Laboratory diagnosis of venereal disease. Public Health Laboratory Service Monograph Series No. 1. HMSO: London. 